\title{
Preface: Special Issue on Optimization Algorithms and Applications
}

\author{
Dong-Dong Ge ${ }^{1} \mathbb{D} \cdot$ Zai-Wen Wen $^{2} \cdot$ Ya-Xiang Yuan ${ }^{3}$
}

Published online: 22 February 2019

(c) Operations Research Society of China, Periodicals Agency of Shanghai University, Science Press, and Springer-Verlag GmbH Germany, part of Springer Nature 2019

Optimization is one of the fundamental and essential components of operations research, a highly interdisciplinary subject. As one of the first researchers of the interior-point methods, Professor Yin-Yu Ye is responsible not only for developing many fundamental results, which have tremendously advanced the optimization theory, but also for enriching the field by applications emerging from statistics, machine learning, signal and imaging processing, communications, computational economics and finance. Computational methods and theory using semidefinite programming have been demonstrated to be helpful for the localization of network sensors. In computational economics, new complexity results have been established for problems related to the computation of an economic equilibrium. We appreciate Ye for his insatiable curiosity, openness to new ideas and a keen interest in the success of young people in our field of operations research.

This issue is a perfect exhibition of his influence and inspiration to many operations research scholars, even to the youngest disciples. The topics in this collection of selected papers encompass a broad area of optimization algorithms and applications, including robust optimization, dynamic pricing, phase retrieval by sensor network localization and asynchronous computation, etc. These papers are summarized as follows.

Zhimin Peng, Yangyang Xu, Ming Yan and Wotao Yin analyzed the convergence of the async-parallel block coordinate update method in a probabilistic way. They showed that the algorithm is guaranteed to converge for smooth problems if the expected

\footnotetext{
$凶 \quad$ Dong-Dong Ge

ge.dongdong@mail.shufe.edu.cn

Zai-Wen Wen

wenzw@pku.edu.cn

Ya-Xiang Yuan

yyx@1sec.cc.ac.cn

1 Shanghai University of Finance and Economics, Shanghai 200433, China

2 Beijing International Center for Mathematical Research, Beijing 100871, China

3 Chinese Academy of Sciences, Beijing 100190, China
} 
delay is finite and for non-smooth problems if the variance of the delay is also finite; moreover, they established sublinear convergence of the method for weakly convex problems and linear convergence for strongly convex ones.

Tao-Ran Fu and Jin-Yan Fan showed that the successive partial-symmetric rank-one approximation algorithm not only exactly recovers the unitary decomposition of the unitarily decomposable conjugate partial-symmetric tensors, but also robustly recovers the unitary decomposition of the underlying complex tensor in the presence of perturbations. They studied the partial-symmetric tensors in the complex domain involving conjugate terms and provided the first theoretical analysis of the exact recovery of these tensors.

Peng-Yu Qian, Zi-Zhuo Wang and Zai-Wen Wen proposed a unified framework for decision making under uncertainty using a composite of risk measures. The framework is based on the composite of an inner risk measure that accounts for the risk of decision if the exact distribution of uncertain model parameters is given, and an outer risk quantifies the risk that occurs when estimating the parameters of distribution. Both theoretical proofs and numerical experiments showed that the paradigms yielded less conservative solutions, yet provide the same degree of probabilistic guarantee.

Xin Chen, Zhen-Yu Hu and Yu-Han Zhang proposed a stochastic differential equation to model the reference price evolution in order to capture the temporal heterogeneity in consumer groups as well as exogenous shocks that affect consumers' memory processes. The corresponding dynamic pricing problem was analyzed using the stochastic optimal control theory. The authors provided an explicit expression for the optimal pricing strategy and the steady state distribution by solving the HamiltonJacobi-Bellman equation.

Sherry Xue-Ying Ni, Man-Chung Yue, Kam-Fung Cheung and Anthony Man-Cho So connected the problem of phase retrieval to the well-studied problem of sensor network localization. A two-stage algorithm that can provably recover the desired signal was developed for phase retrieval. The proposed algorithm improves upon prior approaches in terms of the number of required measurements and the reconstruction time.

John Gunnar Carlsson and Ye Wang used the Wasserstein distance to characterize the ambiguity set of distributions in distributionally robust optimization problems. Their approach can overcome common overestimation when fixing the center of mass and the covariance matrix of the distribution. They also provided a districting strategy for coordinating a fleet of vehicles for the problem of finding a distribution whose highest density region contains as little mass as possible subject to Wasserstein distance constraints.

Qi Qi, Wen-Wei Wang and Ling-Fei Yu considered revenue maximization in viral marketing of competitive and collaborative products through social networks. They studied the efficient allocation problem of the publisher to maximize its revenue. The algorithmic complexity approach was taken for revenue maximization under the optimization models. The NP-hardness was proved, and non-approximability results were obtained for general structures and polynomial time algorithms and applications for special classes of networks were established.

All the papers in this special issue have been peer reviewed to the standard of the journal. Our deep and sincere gratitude goes to all the authors for their timely response 
and careful revisions. They have made tremendous contributions and offered generous support to this issue. We also thank the anonymous referees for their voluntary work and expert reviews.

November 2018 